\title{
ANALYSIS OF NATURAL VARIATION IN OSHKT1;1 GENE SEQUENCE AND GENE EXPRESSION IN RELATION TO SALINITY IN RICE (Oryza sativa L.)
}

\author{
P. T. Do ${ }^{1, *}$, D. H. Nguyen ${ }^{1,2}$ and H. T. Tang ${ }^{3}$ \\ ${ }^{1}$ VNU University of Science, Vietnam National University Hanoi, 334 Nguyen Trai, Thanh Xuan, Hanoi, Vietnam \\ ${ }^{2}$ Hong Duc University, 565 Quang Trung, Dong Ve, Thanh Hoa, Vietnam; ${ }^{3}$ Vietnam National University of Agriculture, \\ Trau Quy, Gia Lam, Hanoi, Vietnam \\ Corresponding Author's E-mail: dothiphuc13380@gmail.com; phucthido@vnu.edu.vn
}

https://doi.org/10.36899/JAPS.2020.1.0019

Published online January 02, 2020

\begin{abstract}
Rice is a main food crop, but sensitive to salinity. The high affinity $\mathrm{K}^{+}$transporters (HKTs) have been proven to be important to salt tolerance in plants. In the current study, the natural variations in gene sequence of $O s H K T 1 ; 1$ were investigated for uncovering the potential allelic variants in salt tolerance in rice. Sequence analysis of OsHKT1;1 gene was conducted in the coding and promoter regions of all investigated rice cultivars. There were nine SNPs in the coding sequences and ten SNPs in the promoter sequences. Amongst nine SNPs found in the coding sequences, four were nonsynonymous (C89T, C280T, T536C, A773G) leading to four substituted amino acids P30L, L94F, F179S, and N258S. In silico analysis revealed no potential effects of the substituted amino acids to protein structure, but caused changes in post-translational modifications. In the promoter sequences, eight out of ten SNPs caused to five additions and six deletions of the cis-regulatory elements, in which some of them were shown to be involved in stress responses. OsHKT1;1 gene expression was analyzed in the roots and leaves in response to different salt concentrations. While the expression of OsHKT1;1 was induced in the leaves at the beginning of salt treatment (day 1 and day 2), it was decreased or unchanged in the roots.
\end{abstract}

Keywords: OsHKT1;1, salinity, rice, polymorphism.

\section{INTRODUCTION}

Salinity is one major environmental problem that reduces agricultural productivity. Salt stress causes serious problems to plants by combining both ionic stress and osmotic stress. High accumulation of toxic elements, such as $\mathrm{Na}^{+}$, leads to toxic to the cell. Therefore, regulation of $\mathrm{Na}^{+}$concentration in the cell plays a key role for survival of plants under saline condition (Tester and Davenport, 2003; Almeida et al., 2017).

High affinity $\mathrm{K}^{+}$transporters (HKTs) are an essential determinant for salt tolerance in plants. Members of HKTs are proposed to protect the leaves from overaccumulation of $\mathrm{Na}^{+}$by mediating xylem $\mathrm{Na}^{+}$ unloading in the roots, phloem $\mathrm{Na}^{+}$loading in the shoots (Berthomieu et al., 2003; Sunarpi et al., 2005; Ren et al., 2005; Byrt et al., 2007; Davenport et al., 2007; Almeida et al., 2017), and by enhancing exclusion of $\mathrm{Na}^{+}$ion from leaf blades during salt stress (Wang et al., 2015; Kobayashi et al., 2017). Overexpression of AtHKT1;1 enhances salt tolerance in rice, and GmHKT1;4overexpressed transgenic tobacco showed improvement of salinity tolerance (Plett et al., 2010; Chen et al., 2014). Cucumber grafted with salt tolerant pumpkin as rootstock showed growth enhancement under salt stress (Sun et al., 2018).
The HKT protein family can be divided in two classes based on structure and transport characteristics. Class 1 HKT transporter has a serine in the first pore domain, forming an S-G-G-G motif, and shows $\mathrm{Na}^{+}$selective transport. Whereas, class 2 members possess a glycine with an G-G-G-G motif, and generally exhibit $\mathrm{Na}^{+}-\mathrm{K}^{+}$co-transport (Mäser et al, 2002; Platten et al., 2006). Rice has nine HKT genes, consisting of OsHKT1;1, OsHKT1;2, OsHKT1;3, OsHKT1;4, OsHKT1;5, OsHKT2;1, OsHKT2;2, OsHKT2;3, and OsHKT2;4. OsHKT1;2 is a pseudo gene in which the stop codon appears inside the gene and OSHKT2;2 is not found in Nipponbare cultivar (Platten et al., 2006). The rice class 1 members, except OsHKT1;2, show permeable to $\mathrm{Na}^{+}$only (Ren et al., 2005; Jabnoune et al., 2009; Suzuki et al., 2016); while class 2 displays diverse transport properties. OsHKT2;1 is either $\mathrm{Na}^{+}-\mathrm{K}^{+}$symport or $\mathrm{Na}^{+}$uniport depending on concentration of external $\mathrm{K}^{+}$ and $\mathrm{Na}^{+}$(Garciadeblas et al., 2003; Horie et al., 2007; Jabnoune et al., 2009). OsHKT2;2 shows $\mathrm{Na}^{+} / \mathrm{K}^{+}$cotransport, while OsHKT2;4 exhibits strong $\mathrm{K}^{+}$ permeability with ability to also transport $\mathrm{Mg}^{2+}$ and $\mathrm{Ca}^{2+}$ (Yao et al., 2010; Horie et al., 2011). The rice HKT genes are found to diversely express (Almeida et al., 2013 and Almeida et al., 2017 and reference herein). Some members of the rice HKT transporter family are 
involved in the salt tolerance mechanism, such as OsHKT1;1, OsHKT1;4, OsHKT1;5 (Ren et al., 2005; Cotsaftis et al., 2012; Wang et al., 2015; Suzuki et al., 2016; Campbell et al., 2017; Kobayashi et al., 2017).

OsHKT1;1 belongs to class 1 of rice HKT family. OsHKT1;1 has been proven to play important roles in salt tolerance of rice by enhancing exclusion of $\mathrm{Na}^{+}$ion from leaf blades. The regulation of $\mathrm{Na}^{+}$ion exclusion from the plant shoot potentially via xylem-tophloem transfer mechanism (Wang et al., 2015). The OsHKT1;1 gene was found to be highly expressed in the vascular tissue of the shoot than those of the root (Jabnoune et al., 2009; Wang et al., 2015). And OsHKT1;1 gene was well expressed at the seedling stage (Campbell et al., 2017). Upon salt stress, OsHKT1;1 expression was increased 3- to 5-fold in the shoots, but not in the roots (Wang et al., 2015). OsHKT1;1 is the causal gene in the RNC4 QTL controlling the root $\mathrm{Na}^{+}$content and $\mathrm{Na}^{+}$to $\mathrm{K}^{+}$ratio (Campbell et al., 2017).

Rice (Oryza sativa) is a main food crop worldwide, but sensitive to salinity. Improving salt tolerance in rice is crucial. Previous works have demonstrated the importance of allelic variations in the sequences of ion transporter genes to salt tolerance in plants. A clear example showing the important role of the allelic variations to salt tolerance found in $O s H K T 1 ; 5$ gene. The nucleotide substitution in the coding sequence that lead the V395L substitution in OsHKT1;5 transporter of the salt tolerant Nona Bokra cultivar compared to the salt sensitive Koshihikari cultivar enhanced $\mathrm{Na}^{+}$transport activity (Ren et al., 2005; Cotsaftis et al., 2012). In other findings, three nucleotide polymorphisms within OsHKT1;1 gene were proposed to associate with modified $\mathrm{Na}^{+}$accumulation in the rice root (Campbell et al., 2017), and two nucleotide substitutions in ZmHKT1;5 gene sequence were proven to associated with salt tolerance in maize (Jiang et al., 2018).

In the current study, the natural variations in the OsHKT1;1 gene sequence were investigated in different rice cultivars. Both the coding sequence and the regulatory upstream sequence of the gene were subjected for studying, and the detected nucleotide polymorphisms were further analyzed to evaluate potential influence on protein characteristics and transcriptional/ translational regulation. In addition, OsHKT1;1 gene expression in response to different salt concentrations was studied in two contrasting rice cultivars.

\section{MATERIALS AND METHODS}

Rice materials and stress treatment: Seeds of nine rice cultivars, including Pokkali, Nipponbare, Cuom-2, Chanh-Trui, IR29, Nuoc-Man-2, Cham-Bien, ChiemRong, Nuoc-Man-1 were kindly provided by Vietnam
National University of Agriculture (Hanoi, Vietnam). Most of the cultivars were found in the coastal area of Vietnam. Pokkali was used as salt tolerant reference cultivar, while Nipponbare and IR29 were considered as salt sensitive reference cultivars. Plant cultivation and salt treatment were performed as described previously in Do et al. (2018).

Salt tolerance evaluation: Rice cultivars were evaluated for salt tolerance using modified standard evaluation score (SES) which is based on visual salt injury of plants at seedling stage (Gregorio et al., 1997; Bado et al., 2016). Three plants per cultivar was used.

Extraction of genomic DNA: The genomic DNA was extracted from the leaf materials using the CTAB method as described previously in Do et al., 2018.

OsHKT1;1 gene amplification using PCR technique: OsHKT1;1 upstream sequence and gene sequence were amplified from DNA template by PCR using specific primers, following by direct sequencing. The list of PCR primers is shown in Table 1 and primer binding sites in the cds and promoter sequences are indicated in Supplementary Fig. S1.

Each PCR mixture contained genomic DNA (about $50 \mathrm{ng}$ ), Dream Taq polymerase buffer $(1 \times)$, $\mathrm{MgCl} 2(1.5 \mathrm{mmol} / \mathrm{L})$, Dream Taq polymerase (1 U), primers $(0.4 \mu \mathrm{mol} / \mathrm{L})$, and dNTPs $(0.2 \mathrm{mmol} / \mathrm{L})$. Thermal cycle condition for each PCR reaction was conducted as following: $95^{\circ} \mathrm{C}$ for $5 \mathrm{~min}, 35$ cycles of denature at $95^{\circ} \mathrm{C}$ for $30 \mathrm{~s}$, annealing for $30 \mathrm{~s}$ and extension at $72^{\circ} \mathrm{C}$ for 2 $\mathrm{min}$, and $72^{\circ} \mathrm{C}$ for $5 \mathrm{~min}$. After purification the products of PCR were sequenced by the First BASE DNA sequencing service (Singapore). The obtained sequences were sent to the GenBank database. The accession numbers for gene sequences containing the coding sequences are: MK541575 (Nipponbare), MK541577 (Nuoc-Man-2), MK541576 (Nuoc-Man-1), MK541582 (Chanh-Trui), MK541578 (Cuom-2), MK541580 (Pokkali), MK541579 (IR29), MK541581 (Chiem-Rong), and MK541583 (Cham-Bien). The accession numbers for the upstream regions of $O s H K T 1 ; 1$ gene are: MK541584 (Nipponbare), MK541585 (Nuoc-Man-2), MK541591 (Nuoc-Man-1), MK541586 (Cuom-2), MK541590 (Chanh-Trui), MK541587 (Pokkali), MK541588 (IR29), MK541592 (Chiem-Rong), and MK541589 (ChamBien).

Sequence analysis: To analyze the nucleotide sequences Multalin webserver (Corpet, 1988) and Bioedit (Hall, 1999) were used. The amino acid sequences were predicted and analyzed by using the Expasy webserver (http://web.expasy.org/translate/). The sequences of OsHKT1;1 of different rice cultivars were compared to Nipponbare cultivar's sequence. 
Table 1. Primers used for PCR amplification of OsHKT1;1.

\begin{tabular}{|c|c|c|c|c|c|c|}
\hline \multirow{3}{*}{$\begin{array}{l} \\
\text { Primer for } \\
\text { gene sequence }\end{array}$} & \multirow{3}{*}{$\begin{array}{l}\text { Primer name } \\
\text { CdsHKT1.1-1 }\end{array}$} & \multicolumn{4}{|c|}{ Sequence } & \multirow{2}{*}{$\begin{array}{l}\begin{array}{l}\text { Amplicon } \\
\text { size (bp) }\end{array} \\
775\end{array}$} \\
\hline & & FW & 5 '- & GGCAGCGTTACACAAGTACCA-3' & $-3^{\prime}$ & \\
\hline & & RV & 5 '- & ACCAACACAGCGCAAGGATT-3' & $-3^{\prime}$ & \\
\hline & \multirow{2}{*}{ CdsHKT1.1-2 } & FW & 5 '- & GATGCTAATGGGAGGAGAGGTG & -3 ' & \multirow[t]{2}{*}{784} \\
\hline & & RV & 5 '- & TTCACCGGCTTGTCTTGTAT & -3 ' & \\
\hline & \multirow[t]{2}{*}{ CdsHKT1.1-3 } & FW & 5 '- & CCTGGGGAGACTGGCTACAA & -3 ' & \multirow[t]{2}{*}{680} \\
\hline & & RV & 5 '- & TGAAGCATCGGAAGGAAGGTA & $-3^{\prime}$ & \\
\hline & \multirow[t]{2}{*}{ CdsHKT1.1-4 } & FW & 5 '- & TGCAGCTTGCTAGGTTTTATCG & -3 ' & \multirow[t]{2}{*}{570} \\
\hline & & RV & 5 '- & TGCTGGTTCAGTGGTTCTTAGTG & $-3^{\prime}$ & \\
\hline & \multirow{2}{*}{ CdsHKT1.1-5 } & FW & 5 '- & CTGATAACCAGCCCTTGACAG & -3 ' & \multirow[t]{2}{*}{688} \\
\hline & & RV & 5 '- & CAATCGTGATTCGGGGTCAATC & $-3^{\prime}$ & \\
\hline \multirow{6}{*}{$\begin{array}{l}\text { Primer for } \\
\text { Upstream } \\
\text { sequence }\end{array}$} & \multirow{2}{*}{ ProHKT1.1-1 } & FW & 5 '- & GGGTGAATTAGACATAACCATAGGA & -3 ' & \multirow[t]{2}{*}{706} \\
\hline & & RV & 5 '- & ACTACGCGTTGGTTTGTGC & -3 ' & \\
\hline & \multirow[t]{2}{*}{ ProHKT1.1-2 } & FW & 5 '- & AGTCCATAGAAAGCAAGCGG & -3 ' & \multirow[t]{2}{*}{721} \\
\hline & & RV & 5 '- & TACTCCGAAAAGGACTCGTGG & $-3^{\prime}$ & \\
\hline & \multirow[t]{2}{*}{ ProHKT1.1-3 } & FW & 5 '- & TGTAGCGACTTCCTTGACTGC & $-3^{\prime}$ & \multirow[t]{2}{*}{578} \\
\hline & & RV & 5'- & GACAGGAACCCACAGAGATGC & $-3^{\prime}$ & \\
\hline
\end{tabular}

Examination of putative changes at protein level: Several bioinformatics tools were used to determine consequent influences of the nucleotide substitutions on variant protein properties. Transmembrane protein structure prediction based on PSIPRED web server (http://bioinf.cs.ucl.ac.uk/psipred/) and UniProt database (https://www.uniprot.org/). The 3D structures of the proteins were predicted by I-TASSER program (Zhang et al., 2008). The UCSF Chimera v1.13.1 was used to visualize the 3-D protein structure (Pettersen et al., 2004). To analyze the protein putative post-translational modifications, the PlantPhos was applied for phosphorylation site prediction (Lee et al., 2011), a webbased server http://csb.cse.yzu.edu.tw/UbiSite/ was used for ubiquitination site analysis, and SUMOplot ${ }^{\mathrm{TM}}$ (http://www.abgent.com/sumoplot) was applied to analyze the possible SUMOylation sites.

Analysis of putative cis- regulatory elements present in OsHKT1;1 promoter: The PLACE database was used to analyze the putative cis-regulatory elements present in the $1.45-\mathrm{kb}$ upstream region from start codon of the gene (Higo et al., 1999).

OsHKT1;1 gene expression by real-time PCR: The leaves and roots of salt-treated and control seedlings were collected at day $1,2,3$, and 7 of salt treatments. The RNA isolation and synthesis of cDNA were performed as described in Do et al., 2018.

The OsHKT1;1 gene expression was analyzed by real-time PCR technique using the following primers: FW 5'- TGCAGTCGTCATGTACCTTCC -3', RW: 5'CGGTAAAATTCCTCCACAGTGC -3'. PCR mixture included $1 \mu \mathrm{L}$ of diluted cDNA, $0.6 \mu \mathrm{L}$ of primer mix $(10 \mu \mathrm{M}), 10 \mu \mathrm{L}$ of SYBR Green Master Mix 2X
(Luminaris Higreen low ROX qPCR master mix, Thermo Fisher Scientific) in a $20 \mu \mathrm{L}$ reaction. The PCR reaction was run as: $95^{\circ} \mathrm{C}$ for $10 \mathrm{~min}$, then 40 cycles of $\left(95^{\circ} \mathrm{C}\right.$ for $15 \mathrm{~s}, 60^{\circ} \mathrm{C}$ for $1 \mathrm{~min}$ ) in ABI Fast 7500 System (Applied Biosystems). Actin1 was used as reference gene (Caldana et. al., 2007) with primers FW: 5'CTCCCCCATGCTATCCTTCG-3', RW: 5'TGAATGAGTAACCACGCTCCG-3'. The specificity of the PCR reaction was assessed by analyzing melting curve and agarose gel electrophoresis. The expression levels of $O S H K T 1 ; 1$ gene were analyzed using the $2^{-\Delta \Delta \mathrm{Ct}}$ method (Livak and Schmittgen, 2001). Two biological replicates and two technical replicates were performed.

\section{RESULTS}

Variation in salinity tolerance among rice cultivars: The nine rice cultivars were cultivated using hydroponic culture system and were treated with salt stress $(100 \mathrm{mM}$ $\mathrm{NaCl}$ ) at the seedling stage. As described in study of Gregorio et al. (1997) the scoring of visual injury symptom at seedling stage is the reliable parameter for distinguish the tolerant, moderate, and susceptible groups in rice. In our previous study, the seven out of nine rice cultivars were classified into different salt tolerant levels based on the leaf scoring (Do et al., 2018). In which, Pokkali, Chanh-Trui, Nuoc-Man-1, Cuom-2 were considered as salt tolerant; Nuoc-Man-2, Chiem-Rong were classified moderate; and Nipponbare was defined as sensitive. In this study, two rice cultivars, IR29 and Cham-Bien, were included. The scoring of Cham-Bien was 3.0 and IR29 was 9.0. Thus, Cham-Bien was 
classified as tolerant and IR29 was considered highly susceptible.

Variations in the $\boldsymbol{O S H K T 1 ; 1}$ coding sequence: In order to identify the polymorphisms in the OsHKT1;1 gene sequence, the gene was firstly amplified by the PCR technique using different primer pairs (Table 1). After sequencing, only the coding sequence of the gene was further analyzed. Using Nipponbare culvitivar gene sequence as reference, sequencing analysis could allow to identify nine nucleotide substitutions in the OsHKT1;1 coding sequence (Fig 1). The alignment of protein sequences is shown in Supplementary Fig. S2.

Amongst nine detected SNPs, four were nonsynonymous (C89T, C280T, T536C, A773G) and other five were synonymous substitutions (T189C, T225C, A321G, A885C, C912T). The non-synonymous C89T, C280T, T536C, A773G caused the amino acid changes, including P30L, L94F, F179S, N258S, respectively (Table 2).

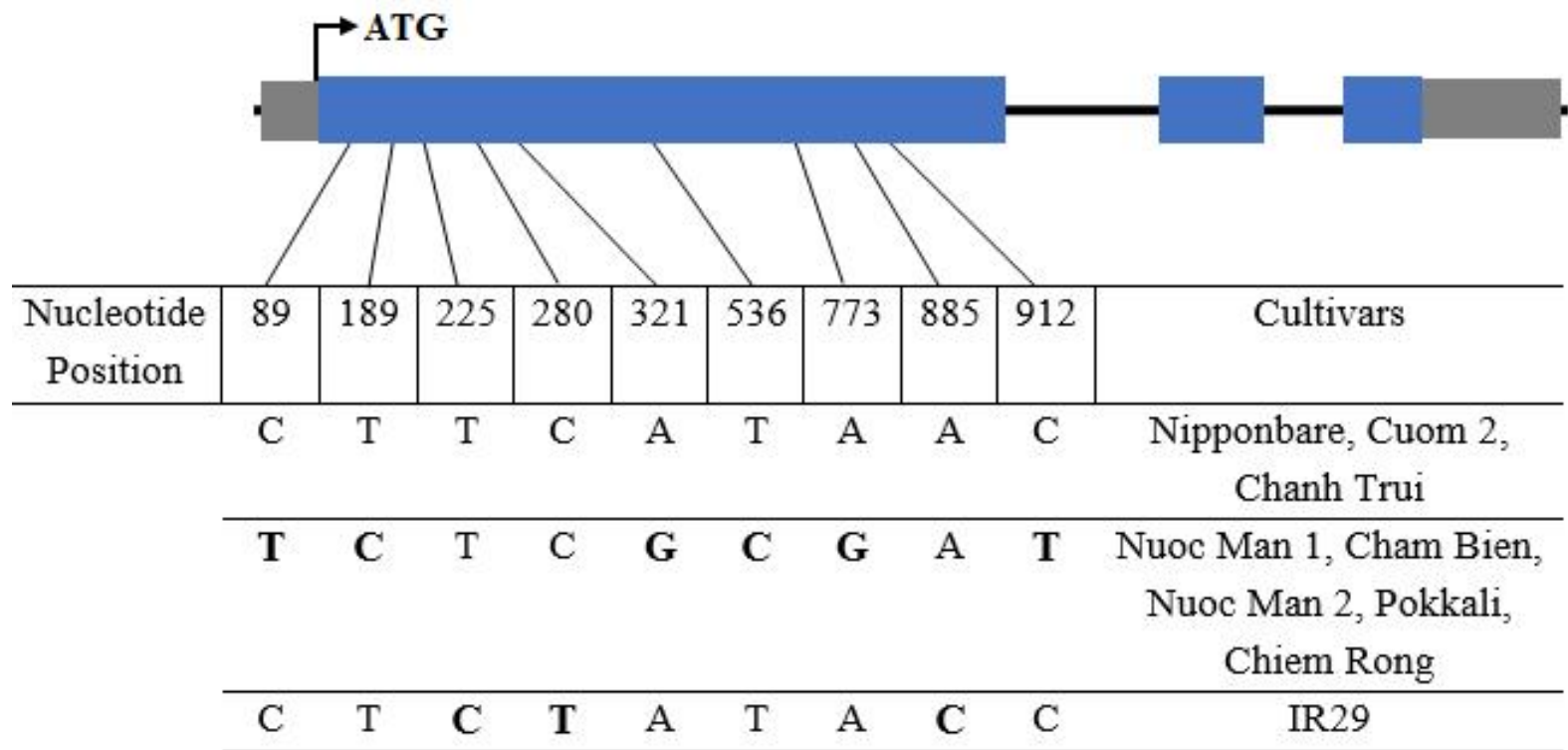

Figure 1. Allelic polymorphisms in the OsHKT1;1 coding region. Bold letters indicate the nucleotide polymorphisms.

Table 2. Putative effect of nucleotide polymorphisms present in the OsHKT1;1 coding region.

\begin{tabular}{|c|c|c|c|c|}
\hline Position & \multicolumn{2}{|c|}{ Polymorphic type } & \multirow{2}{*}{$\begin{array}{c}\text { Effect on } \\
\text { post-translational } \\
\text { modification } \\
\end{array}$} & Cultivars \\
\hline 89 & $\mathrm{C} / \mathrm{T}$ & $\begin{array}{c}\text { Non-Synonymous } \\
\text { (P30L) }\end{array}$ & & \multirow{3}{*}{$\begin{array}{l}\text { Chiem-Rong, Cham-Bien, Nuoc- } \\
\text { Man-2, Pokkali, Nuoc-Man-1 }\end{array}$} \\
\hline 189 & $\mathrm{~T} / \mathrm{C}$ & Synonymous & & \\
\hline 225 & $\mathrm{~T} / \mathrm{C}$ & Synonymous & & \\
\hline 280 & $\mathrm{C} / \mathrm{T}$ & $\begin{array}{l}\text { Non-Synonymous } \\
(\mathbf{L 9 4 F})\end{array}$ & Sumoylation at $92 \mathrm{~K}$ & \multirow[t]{2}{*}{ IR29 } \\
\hline 321 & $\mathrm{~A} / \mathrm{G}$ & Synonymous & & \\
\hline 536 & $\mathrm{~T} / \mathrm{C}$ & $\begin{array}{l}\text { Non-Synonymous } \\
\text { (F179S) }\end{array}$ & $\begin{array}{c}\text { Phosphorylation at } 179 \mathrm{~S}, \\
181 \mathrm{~S}\end{array}$ & \multirow{2}{*}{$\begin{array}{l}\text { Chiem-Rong, Cham-Bien, Nuoc- } \\
\text { Man-2, Pokkali, Nuoc-Man-1 }\end{array}$} \\
\hline 773 & $\mathrm{~A} / \mathrm{G}$ & $\begin{array}{l}\text { Non-Synonymous } \\
\text { (N258S) }\end{array}$ & $\begin{array}{c}\text { Phosphorylation at } 254 \mathrm{~S} \\
\text { Ubiquitination at K255 }\end{array}$ & \\
\hline 885 & $\mathrm{~A} / \mathrm{C}$ & Synonymous & & \multirow{2}{*}{$\begin{array}{c}\text { IR29 } \\
\text { Chiem-Rong, Cham-Bien, Nuoc- } \\
\text { Man-2, Pokkali, Nuoc-Man-1 }\end{array}$} \\
\hline 912 & $\mathrm{C} / \mathrm{T}$ & Synonymous & & \\
\hline
\end{tabular}

To elucidate the putative influence of nucleotide variations on protein characteristics, the structural model of OsHKT1;1 protein was predicted and the positions of these non-synonymous substitutions on protein domains were analyzed. As shown in Fig 2B, the OsHKT1;1 3D model has the presence of three glycine (Gly 275, Gly 
399, and Gly 501) and one serine (Ser 119), forming a selectivity filter pore. OsHKT1;1 possesses eight transmembrane domains (TMDs) with four pore loops A, $\mathrm{B}, \mathrm{C}$, and D (Fig 2A). The substituted amino acid P30L is located in the N-ternimal cytosolic region, L94F and N258S lay in the outer loops between TMD 1 and TMD2 and between TMD 3 and TMD 4, respectively, and F179S is positioned in inner loop between TMD 2 and TMD 3 (Fig 2). Relied on the predicted structure, these substitutions unlikely interfere the $\mathrm{Na}^{+}$transport activity of the variant OsHKT1;1 transporter.
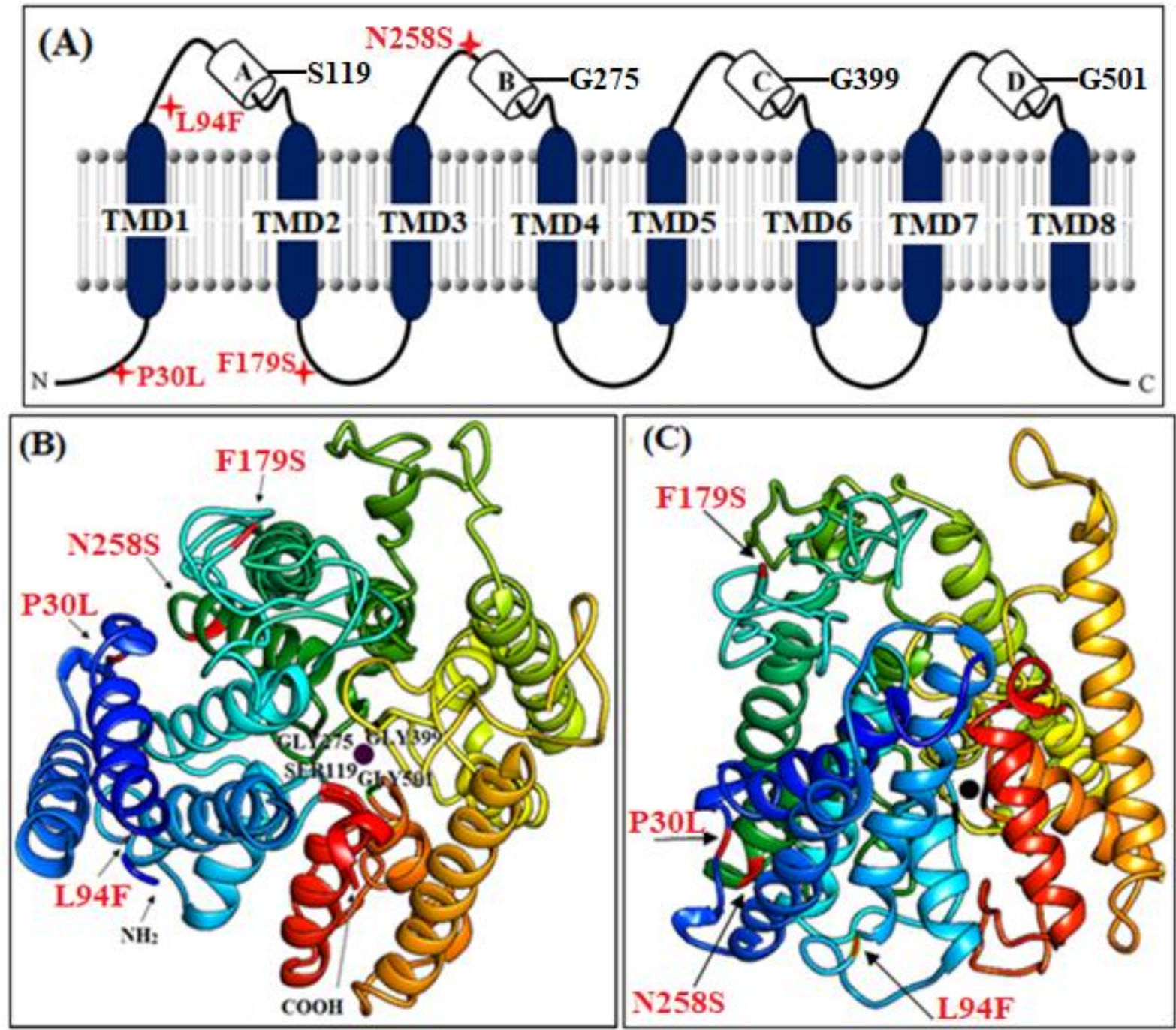

Figure 2. Modelling of OsHKT1;1 protein structure. A: The 2D model with motif of four transmembrane-pore looptransmembrane domain. Asterisks indicate positions of substituted amino acids. B: 3D model of the OsHKT1;1 transporter from the top/from the side. The Ser-Gly-Gly-Gly motif forms the ion filter pore. The black dot at center of the pore represents $\mathrm{Na}^{+}$ion. $\mathrm{C}$ : Visualization from the side of protein model, showing positions of four amino acid variants.

Additionally, the consequent effects of the amino acids on putative post-translational modifications, such as ubiquitination, SUMOylation and phosphorylation of OsHKT1;1 protein were analyzed. The substitutions of phenylalanine to serine (F179S), and asparagine to serine (N258S) provided two serine residues which were predicted to be phosphorylated. The substitutions of phenylalanine to serine (F179S) increased the probability of phosphorylation at 181-serine (Table 2). The N258S substitution also increased the probability of ubiquitination at 255-lysine residue. And the substitution of leucine to phenylalanine (L94F) increased the SUMOyl probability of 92-lysine residue. The putative phosphorylation and ubiquitination were found in cultivars Chiem-Rong, Cham-Bien, Nuoc-Man-2, Pokkali, Nuoc-Man-1; while the predicted SUMOylated 
Table 3. Polymorphisms in the OsHKT1;1 upstream sequence

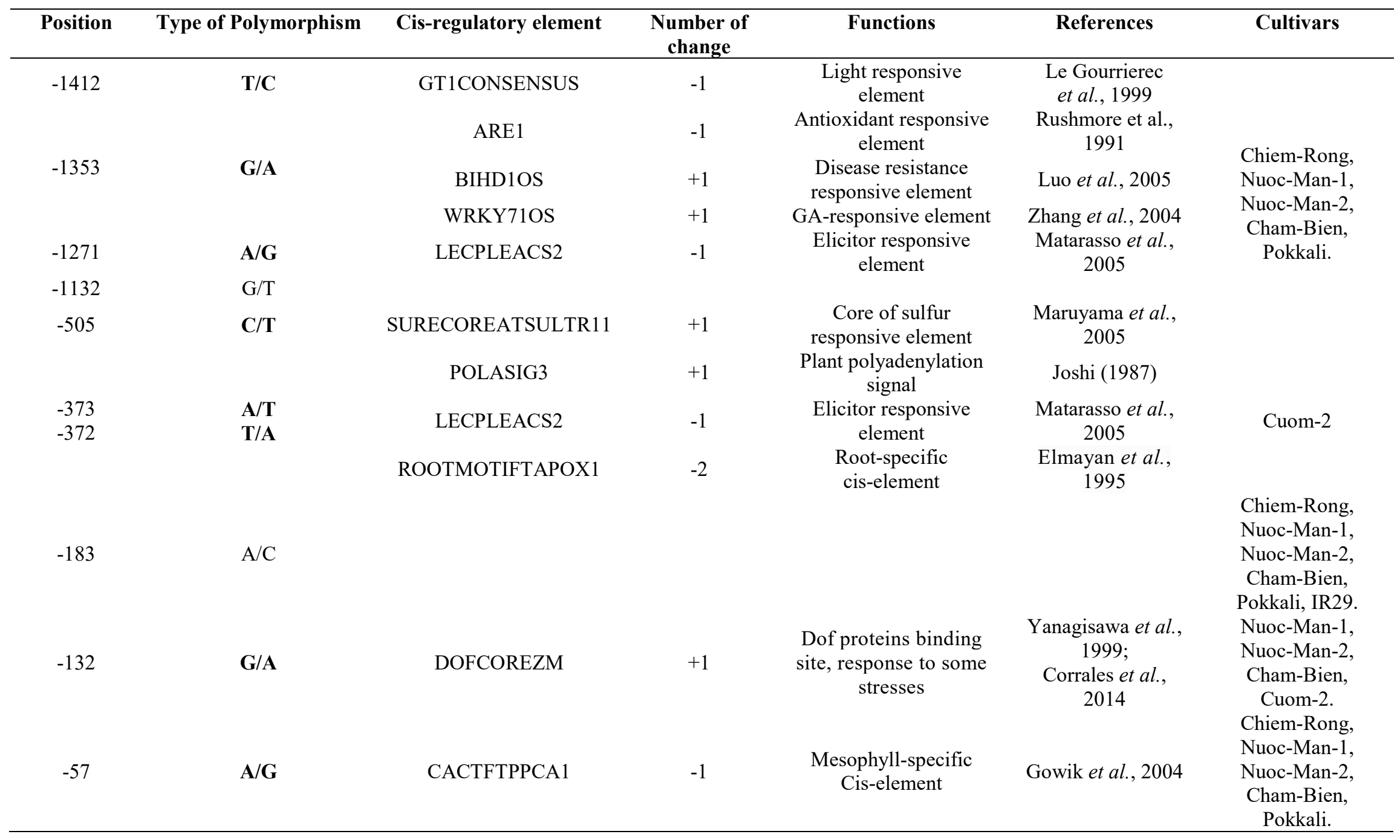


L94F was observed in only cultivar IR29 (Table 2). If these predicted amino acid residues are indeed sites for post-translational modifications, they might alter the functions of the variant OsHKT1;1 transporter.

Variations in the OsHKT1;1 upstream region: The OsHKT1;1 upstream sequence was amplified using PCR technique. Different primer pairs were designed to amplify three amplicons which together covering the 1.45 $\mathrm{kb}$ upstream sequence of $O_{S H K T 1 ; 1}$ gene. To elucidate the nucleotide variation in this regulatory region of OsHKT1;1 gene, the amplified DNA fragments were then sequenced. By comparing the nucleotide sequences, ten nucleotide substitutions (T-1412C, G-1353A, A-1271G, G-1132T, C-505T, A-373T, T-372A, A-183C, G-132A, A-57G) were identified (Table 3). The alignment of nucleotide sequences is shown in Supplementary Fig.S3. Since the nucleotide substitutions can cause changes in cis-regulatory elements, which in turn affect the regulatory function of promoter, the putative cisregulatory elements in OsHKT1;1 promoter were predicted using PLACE database. The result showed that 8 out of 10 nucleotide substitutions led to cis-regulotary elements change (Table 3). Amongst them, some of the changed cis- regularoty elements are involved in stress responses, such as SURECOREATSULTR11, GT1CONSENSUS, DOFCOREZM, and WRKY71OS. SURECOREATSULTR11 is responsive to sulfur deficiency conditions in Arabidopsis (MaruyamaNakashita et al., 2005); GT1CONSENSUS is known as
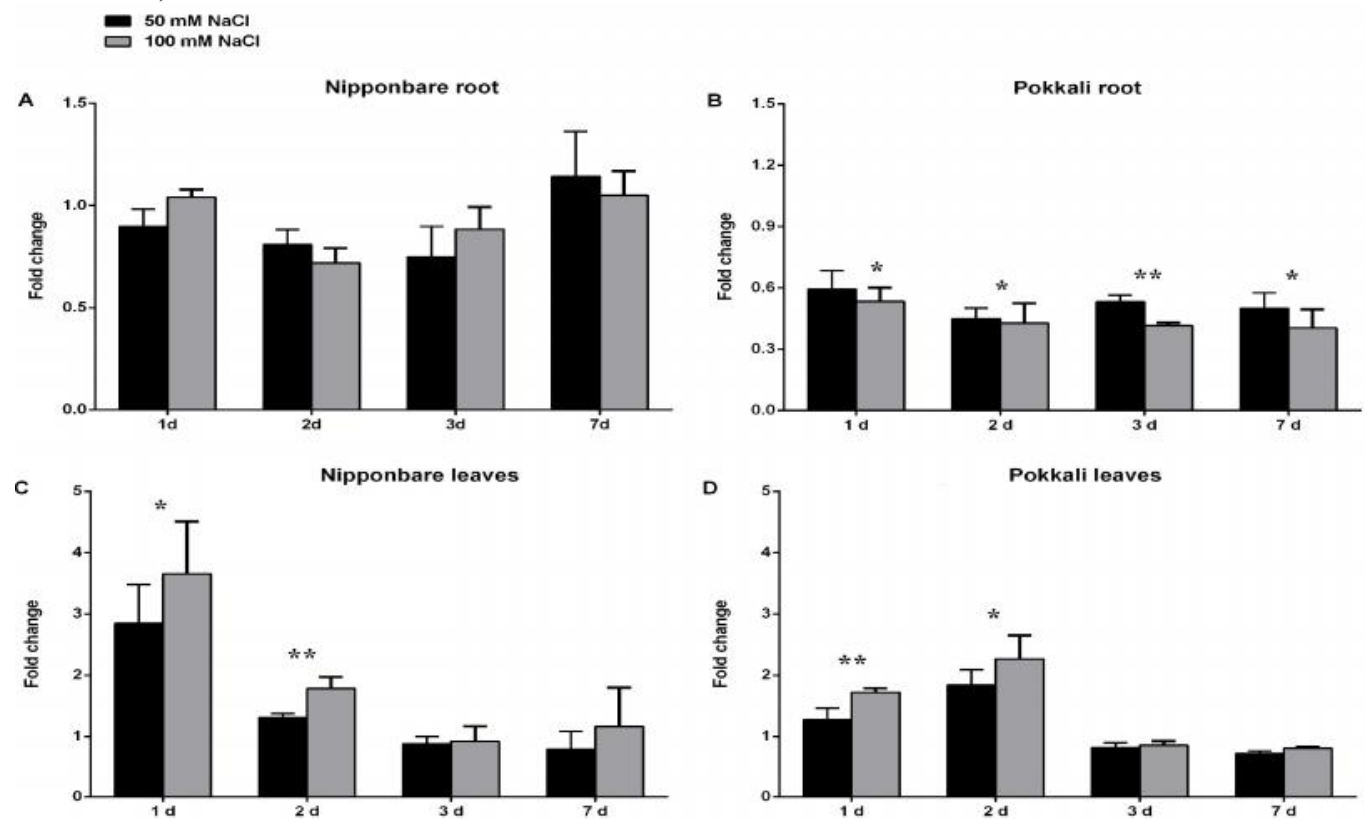

Figure 3. OsHKT1;1 expression profile under different salt conditions. The expression level of $O s H K T 1 ; 1$ gene was computed with the expression level of actin 1 . Fold changes were calculated by using $\Delta \Delta \mathrm{Ct}$ method (Livak and Schmittgen, 2001). Values represent the mean and standard deviation of two biological replicates with two technical replicates per biological experiment $(n=4)$. The asterisk indicates the significant difference; in which one asterisk shows significantly differences between salt and control conditions, two asterisks means significantly differences between salt and control conditions as well as between two salt conditions. 


\section{DISCUSSION}

Though rice is an important food crop worldwide, it is particularly sensitive to soil salinity. Exploring natural genetic resources to find novel alleles for salt tolerance improvement of crop plants is of importance. In the current work, the natural variation in the sequence and the expression of OsHKT1;1 gene was investigated. We could identify in total nine nucleotide variations in the coding sequence and ten ones in the promoter sequence of the OsHKT1;1 (Fig 1, Table 3).

In the coding sequence, four nonsynonymous SNPs were detected, including C89T, C280T, T536C, A773G, which caused the amino acid changes; P30L, L94F, F179S, and N258S, respectively (Table 2). The three substituted amino acids (P30L, F179S, N258S) were observed in the salt-tolerant cultivars Cham-Bien, Chiem-Rong, Nuoc-Man-1, Pokkali, Nuoc-Man-2, while L94F was found in the salt-sensitive cultivar IR29. In order to predict the putative effects of these allelic variants, the protein structure model of OsHKT1;1 was conducted. Based on molecular models of OsHKT1; 1 , the $\mathrm{P} 30 \mathrm{~L}$ is located in the N-ternimal cytosolic region, L94F and N258S are laid in the outer loops and F179S is positioned in inner loop (Fig 2). The selective filter pore with an S-G-G-G motif was found with presence of three 3 glycine residues (Gly 275, Gly 399, and Gly 501) and one serine residue (Ser 119). This predicted S-G-G-G motif of OsHKT1;1 is in agreement with report of high selectivity for $\mathrm{Na}^{+}$of OsHKT1;1 transporter (Jabnoune et al., 2009). Because four amino acid variants are not located in or close to the filter pore of OsHKT1;1 transporter, they are unlikely to have a direct influence in $\mathrm{Na}^{+}$transport of the variant OsHKT1;1 protein However, in the previous study of Campbell et al. (2017), the three amino acid variants (P30L, F179S, N258S) were described to associate with modified $\mathrm{Na}^{+}$accumulation in the roots and responsible for higher $\mathrm{Na}^{+}$transport activity. We next thought that the effect of these variants on the OsHKT1;1 transporter's functions might be at post-translational regulations. Thus, some posttranslational modifications were analyzed using available bioinformatics tools. The results showed that the F179S, N258S alterations could affect the putative phosphorylation of serine residues at position 179 and 258 of OsHKT1;1 protein. The F179S substitution not only was predicted to be phosphorylated serine 179 , but also increased the probability of phosphorylation of serine 181. Furthermore, N258S substitution was predicted to enhance the probability of ubiquitination of lysine residue at position 255 and L94F alteration increased the sumoylation probability of lysine 92 (Table 2 ). It is known that post-translational modifications are involved in the process of activation or suppression of the protein's activity (Friso and Wijk, 2015; Barber and
Rinehart, 2018). The post-translational modifications are important factors that can be regulated to influence salinity tolerance in plants (Roy et al., 2014). Salt overly sensitive 1 (SOS1), an $\mathrm{Na}^{+} / \mathrm{H}^{+}$antiporter, is activated through a protein phosphorylation process by $\mathrm{SCaBP} 8$ SOS2 (Quan et al., 2007) or by SOS2-SOS3 complex (Qiu et al., 2002; Quintero et al., 2011). SOS1 functions in $\mathrm{Na}^{+}$extrusion and hence plays roles in plant salt tolerance (Shi et al., 2000; Olías et al., 2009). The NHX antiporters are also the key determinants of salt tolerance in plants, which are involved in the $\mathrm{Na}^{+}$sequestration into vacuole, thereby maintaining the ion homeostasis (Rodríguez-Rosales et al., 2009). Phospho-proteomic studies of rice plasma membrane and vacuolar membrane indicated that the NHXs are regulated by phosphorylation (Whiteman et al., 2008). In the study of Negrão et al. (2013), a substitution of serine 477 to asparagine $(\mathrm{S} 477 \mathrm{~N})$ in OsNHX1 caused loss of a putative phosphorylation site, and therefore may affect the regulation of this transporter, consequently interfering its function. The reversible conjugation of the SUMO to target protein (sumoylation) may attenuate degradation of target protein (Miura et. al., 2007). Srivastava et. al. (2016) reported that "the control of SUMOylation by OsOTS1 affects salt tolerance in rice". The posttranslational regulations is are of complexity, allowing the organism to smoothly response to environmental changes. For example, in yeast, nitrate transporter (YNT1) transports nitrate and nitrite into the cell. But, adding glutamine to cells grown in nitrate causes a rapid loss of YNT1 activity. This phenomenon is due to the post-translational regulation. When suppling the cells with low nitrate concentration, YNT1 is phosphorylated, preventing YNT1 delivery to vacuole and also decreases ubiquitination. Thus, YNT1 accumulates at plasma membrane, allowing yeast to efficiently use nitrate. When the cells are transferred to medium with glutamine, YNT1 is dephosphorylated, and ubiquitinated. It is then delivered to vacuole for degradation (Navarro et al., 2006; Navarro et al., 2008). Although there are very few experimental reports about the post-translational regulation of HKT- transporter members, this is an interesting possibility to consider. In the current study, the two nonsynonymous (F179S, N258S) of OsHKT1;1 caused the post-translational modifications (phosphorylation, ubiquitination) were observed in salttolerant cultivars Cham-Bien, Chiem-Rong, Nuoc-Man-1, Pokkali, Nuoc-Man-2; while the nonsynonymous L94F leads to sumoylation modification which was found only in salt-sensitive cultivar IR29. We assume that the posttranslational modifications might play a role in regulation of OsHKT1;1 activity, the difference in post-translational regulation might explain the formation of salt-tolerant or salt-intolerant phenotypes. In addition, one possibility for the difference in salt tolerance of rice plants is the expression level of $O s H K T 1 ; 1$ gene. Therefore, we next 
examined the relative expression of OsHKT1;1 gene under different salinity conditions using two contrasting rice genotypes, Pokkali and Nipponbare.

In our study, the expression of $O s H K T 1 ; 1$ was upregulated upon salt stress in the leaves of both rice cultivars, but not in the roots. In the roots, OsHKT1;1 expression level was decreased in Pokkali, but remained unchanged in Nipponbare (Fig. 3). In the previous work, OsHKT1;1 gene expression was reported to induce 3- to 5 - fold by salt stress in the shoots, but not in the roots of Nipponbare cultivar (Wang et al., 2015). Thus, the expression change pattern of OsHKT1;1 observed in Nipponbare cultivar in our study is in agreement with the results of Wang et al., 2015. However, Garciatablas et al., 2003 reported that the OsHKT1;1 (OsHKT4) expression was marginally changed upon stress conditions. The differences in expression pattern of OsHKT1;1 in the work of Garciatablas et al. (2003) might come from the experimental design where a combination of different concentrations of $\mathrm{K}^{+}$and $\mathrm{Na}^{+}$ was used instead of only $\mathrm{Na}^{+}$as in our study. OsHKT1;1 is known to functions in regulating $\mathrm{Na}^{+}$exclusion from the shoot of rice (Wang et al., 2015). Thus, the upregulation of $O s H K T 1 ; 1$ expression in the leaves under salt stress of two rice cultivars might help to exclude the $\mathrm{Na}^{+}$from the leaves in response to salt treatment. If indeed this transporter mainly functions in the shoot tissue of rice plants, the regulation of OsHKT1;1 at transcriptional level might be not different between Pokkali and Nipponbare in response to salt stress as the expression pattern in the shoots of both cultivars was similar. Upon salt stress, both rice cultivars showed upregulation of $O s H K T 1 ; 1$ gene expression in the shoots.

The upstream sequence of the gene with presence of cis- regulatory elements plays the key roles in regulating gene expression (Mariño-Ramírez et al., 2009). In the upstream region of OsHKT1;1 gene, we could detect in total ten nucleotide substitutions, in which eight caused change in cis-regulatory elements. Amongst these changed cis-elements, some are stress responsive elements, including SURECOREATSULTR11 (sulfur responsive), GT1CONSENSUS (light responsive), DOFCOREZM (salt responsive), and WRKY71OS (GA responsive). The addition of one salt-stress responsive cis-element, DOFCOREZM, was observed in salttolerant cultivars, such as Chiem-Rong, Nuoc-Man-1, Nuoc-Man-2, Cham-Bien, and Pokkali (Table 3). However, the DOFCOREZM cis-element present in high number (16) at promoter region of OsHKT1;1 gene (Supplementary Table 1). Thus, it might unlikely that one addition in this cis-element influences gene expression under salt stress, but further experiment should be performed to validate.

In conclusion, we could detect nine SNPs in the coding sequence, and ten SNPs in the upstream sequence of the OsHKT1;1 gene. In silico analysis showed that the
SNPs in the coding sequence caused no effect on protein structure, but affected on post-translational modifications of protein. The SNPs in the upstream sequence of the gene led to changes in several cis-regulatory elements, in which some are involved in abiotic stress response. OsHKT1;1 gene expression in response to different salt stress was upregulated in the leaves, but not in the roots.

Acknowledgments: This research is funded by Vietnam National Foundation for Science and Technology Development (NAFOSTED) under the grant number 106NN.02-2013.47 for Phuc Thi Do.

\section{REFERENCES}

Almeida, P., D. Katschnig, and A.H. Boer (2013). HKT trasporter- state of the art. Int. J. Mol. Sci. 14: 20359-20385.

Almeida, D.M., M.M. Oliveira, and N.J.M. Saibo (2017). Regulation of $\mathrm{Na}+$ and $\mathrm{K}+$ homeostasis in plants: towards improved salt stress tolerance in crop plants. Genet. Mol. Biol. 40: 326-345.

Bado, S., B.P. Forster, A.M.A. Ghanim, J. JankowiczCieslak, G. Berthold, and L. Luxiang (2016). Protocols for pre-field screening of mutants for salt tolerance in rice, wheat and barley. Springer International Publishing AG Switzerland. ISBN 978-3-319-26590-2.

Barber, K.W., and J. Rinehart (2018). The ABCs of PTMs. Nat. Chem. Biol. 14(3): 188-192.

Berthomieu, P., G. Conejero, A. Nublat, W.J. Brackenbury, C. Lambert, C. Savio, N. Uozumi, S. Oiki, K. Yamada, F. Cellier, F. Gosti, T. Simonneau, P.A. Essah, M. Tester, A.A. Véry, H. Sentenac, and F. Casse (2003). Functional analysis of AtHKT1 in Arabidopsis shows that $\mathrm{Na}^{+}$recirculation by the phloem is crucial for salt tolerance. EMBO.J. 22: 2004-2014.

Byrt, C.S., J.D. Platten, W. Spielmeyer, R.A. James, E.S. Lagudah, E.S. Dennis, M. Tester, and R. Munns (2007). HKT1;5-like cation transporters linked to $\mathrm{Na}^{+}$exclusion loci in wheat, Nax2 and Knal. Plant Physiol. 143: 1918-1928.

Caldana, C., W.R. Scheible, B. Mueller-Roeber, and S. Ruzicic (2007). A quantitative RT-PCR platform for high-throughput expression profiling of 2500 rice transcription factors. Plant Methods. 3:7.

Campbell, M.T., N. Bandillo, F.R.A. Al Shiblawi, S. Sharma, K. Liu, Q. Du, A.J. Schmitz, C. Zhang, V. Anne-Alienor, A.J. Lorenz, and H. Walia (2017). Allelic variants of OsHKT1;1 underlie the divergence between indica and japonica subspecies of rice (Oryza sativa) for root sodium content. PloS. Genet. 13.

Chen, H., X. Chen, H. Gu, B. Wu, H. Zhang, X. Yuan, and X. Cui (2014). GmHKT1;4, a novel soybean 
gene regulating $\mathrm{Na}+/ \mathrm{K}+$ ratio in roots enhances salt tolerance in transgenic plants. Plant Growth. Regul. 73: 299-308.

Corpet, F. (1998). Multiple sequence alignment with hierarchical clustering. Nucleic Acids. Res. 16 (22): 10881-10890.

Corrales, A. R., S. G. Nebauer, L. Carrillo, P. FernandezNohales, J. Marques, B. Renau-Morata, A. Granell, S. Pollmann, J. Vicente-Carbajosa, and S. V. Molina (2014). Characterization of tomato cycling Dof factors reveals conserved and new functions in the control of flowering time and abiotic stress responses. J. Exp. Bot. 65: 9951012.

Cotsaftis O., D. Plett, N. Shirley, M. Tester, and M. Hrmova (2012). A two-staged model of $\mathrm{Na}^{+}$ exclusion in rice explained by $3 \mathrm{D}$ modeling of HKT transporters and alternative splicing. PloS. ONE. 7 (7).

Davenport, R.J., A. Muñoz-Mayor, D. Jha, P.A. Essah, A. Rus, and M. Tester (2007). The $\mathrm{Na}+$ transporter AtHKT1;1 controls retrieval of $\mathrm{Na}+$ from the xylem in Arabidopsis. Plant Cell. Environ. 30: 497-507.

Do, P.T., Y.H. Hoang, M.Q Le, H.T. Tang, and D.H. Nguyen (2018). OsHKT1;3 gene sequence polymorphisms and expression profile in rice (Oryza sativa L.). Afr. J. Agric. Res. 13(46): 2659-2667.

Elmayan, T., and M. Tepfer (1995). Evaluation in tobacco of the organ specificity and strength of the rolD promoter, domain $\mathrm{A}$ of the $35 \mathrm{~S}$ promoter and the $35 \mathrm{~S} 2$ promoter. Transgenic Res. 4: 388-396.

Friso, G., and K.J.v. Wijk (2015). Posttranslational protein modifications in plant metabolism. Plant Physiol. 169(3): 1469-1487.

Garciadeblás, B., M.E. Senn, M.A. Banuelos, and A. Rodriguez-Navarro (2003). Sodium transport and HKT transporters: The rice model. Plant. J. 34: 788-801.

Gowik, U., J. Burscheidt, M. Akyildiz, U. Schlue, M. Koczor, M. Streubel, and P. Westhoff. (2004) cis- Regulatory elements for mesophyll-specific gene expression in the $\mathrm{C} 4$ plant Flaveria trinervia, the promoter of the $\mathrm{C} 4$ phosphoenolpyruvate carboxylase gene. Plant Cell. 16(5):1077-90.

Gregorio, G.B., D. Senadhira, and R.D. Mendoza. (1997). Screening rice for salinity tolerance. IRRI Discussion paper series no. 22, IRRI, Manila. 30 p.

Hall, T.A (1999). BioEdit: A user-friendly biological sequence alignment editor and analysis program for Windows 95/98/NT. Nucleic Acids. Symp. Ser., 41: 95-98.
Higo, K., Y. Ugawa, M. Iwamoto, and T. Korenaga (1999). Plant cis-acting regulatory DNA elements (PLACE) database: 1999. Nucleic Acids. Res. 27(1): 297-300.

Horie, T., A. Costa, T.H. Kim, M.J. Han, R. Horie, H.Y. Leung, A. Miyao, H. Hirochika, G. An, and J.I. Schroeder (2007). Rice OsHKT2;1 transporter mediates large $\mathrm{Na}^{+}$influx component into $\mathrm{K}^{+}$starved roots for growth. EMBO. J. 20:30033014.

Horie, T., D.E. Brodsky, A. Costa, T. Kaneko, F. Lo Schiavo, M. Katsuhara, and J.I. Schroeder (2011). $\mathrm{K}^{+}$transport by the OsHKT2;4 transporter from rice with atypical $\mathrm{Na}^{+}$transport properties and competition in permeation of $\mathrm{K}^{+}$ over $\mathrm{Mg}^{2+}$ and $\mathrm{Ca}^{2+}$ ions. Plant Physiol. 156: 1493-1507.

Jabnoune, M., S. Espeout, D. Mieulet, C. Fizames, J.L. Verdeil, G. Conéjéro, A. Rodríguez-Navarro, H. Sentenac, E. Guiderdoni, C. Abdelly, and A.A. Véry (2009). Diversity in expression patterns and functional properties in the rice HKT transporter family. Plant Physiol. 150: 19551971.

Jiang, Z., G. Song, X. Shan, Z. Wei, Y. Liu, C. Jiang, Y. Jiang, F. Jin, and Y. Li (2018). Association Analysis and Identification of ZmHKT1;5 Variation with Salt-Stress Tolerance. Front Plant. Sci. 9: 1485.

Joshi, C. P. (1987). Putative polyadenylation signals in nuclear genes of higher plants: a compilation and analysis. Nucleic Acids. Res. 15(23): $9627-$ 40.

Kobayashi, N.I., N. Yamaji, H. Yamamoto, K. Okubo, H. Ueno, A. Costa, K. Tanoi, H. Matsumura, M. Fujii-Kashino, T. Horiuchi, M.A. Nayef, S. Shabala, G. An, J.F. Ma, and T. Horie (2017). OsHKT1;5 mediates $\mathrm{Na}+$ exclusion in the vasculature to protect leaf blades and reproductive tissues from salt toxicity in rice. Plant. J. 91(4): 657-670.

Le Gourrierec, J., Y. Li, and D. Zhou (1999). Transcriptional activation by Arabidopsis GT-1 may be through interaction with TFIIA-TBPTATA complex. Plant. J. 18: 663-668.

Lee, T.Y., N.A. Bretana, and C.T. Lu (2011). PlantPhos: using maximal dependence decomposition to identify plant phosphorylation sites with substrate site specificity. BMC Bioinformatics. 12: 261 .

Livak, K.J., and T.D. Schmittgen (2001). Analysis of relative gene expression data using real-time quantitative PCR and the 2- $\Delta C t$ method. Methods. 25: 402-408. 
Luo, H., F. Song, R. M. Goodman, and Z. Zheng (2005). Up-regulation of OsBIHD1, a rice gene encoding BELL homeodomain transcriptional factor, in disease resistance responses. Plant. Biol. 7: 459-468.

Mariño-Ramírez, L., K. Tharakaraman, O. Bodenreider, J. Spouge, and D. Landsman (2009). Identification of cis-regulatory elements in gene co-expression networks using A-GLAM. Methods. Mol. Biol (Clifton, N.J.). 541: 1-22.

Maruyama-Nakashita, A., Y. Nakamura, A. Watanabe-Takahashi, E. Inoue, T. Yamaya, and H. Takahashi (2005). Identification of a novel cis-acting element conferring sulfur deficiency response in Arabidopsis roots. Plant J. 42: 305314.

Mäser, P., Y. Hosoo, S. Goshima, T. Horie, B. Eckelman, K. Yamada, K. Yoshida,E.P. Bakker, A. Shinmyo, S. Oiki, J.I. Schroeder, and N. Uozumi (2002).Glycine residues in potassium channellike selectivity filters determine potassiumselectivity in four-loop-per-subunit HKT transporters from plants. Proc. Natl. Acad.Sci. U.S.A., 99: 6428-6433.

Matarasso, N., S. Schuster, and A. Avni (2005). A novel plant cysteine protease has a dual function as a regulator of 1-aminocyclopropane-1-carboxylic acid synthase gene expression. Plant Cell. 17: 1205-1216.

Miura, K., J.B. Jin, and P.M. Hasegawa (2007). Sumoylation, a post-translational regulatory process in plants. Curr. Opin. Plant Biol. 10(5): 495-502.

Navarro, F.J., F. Machín, Y. Martín, and J.M. Siverio (2006). Down-regulation of eukaryotic nitrate transporter by nitrogen-dependent ubiquitinylation. J. Biol. Chem. 281: 1326813274.

Navarro, F.J., Y. Martín, and J.M. Siverio (2008). Phosphorylation of the yeast nitrate transporter Ynt1 is essential for delivery to the plasma membrane during nitrogen limitation. J. Biol. Chem. 283: 31208-31217.

Negrão. S., M.C. Almadanim, I.S. Pires, I.A. Abreu, J. Maroco, B. Courtois, G.B. Gregorio, K.L. McNally, and M.M. Oliveira (2013). New allelic variants found in key rice salt-tolerance genes: An association study. Plant Biotechnol. J. 11: 87-100.

Olías, R., Z. Eljakaoui, J. Li, P.A. De Morales, M.C. Marín-Manzano, J.M. Pardo, A. Belver (2009). The plasma membrane $\mathrm{Na}+/ \mathrm{H}+$ antiporter SOS 1 is essential for salt tolerance in tomato and affects the partitioning of $\mathrm{Na}+$ between plant organs. Plant Cell Environ. 32: 904-916.
Platten, J.D., O. Cotsaftis, P. Berthomieu, H. Bohnert, R.J. Davenport, D.J. Fairbairn, T. Horie, R.A. Leigh, H.X. Lin, S. Luan, P. Mäser, O. Pantoja, A. Rodríguez-Navarro, D.P. Schachtman, J.I. Schroeder, H. Sentenac, N. Uozumi, A.A. Véry, J.K. Zhu, E. S. Dennis, and M. Tester (2006). Nomenclature for HKT transporters, key determinants of plant salinity tolerance. Trends Plant Sci. 11: 372-374.

Pettersen, E. F., T. D. Goddard, C. C. Huang, G. S. Couch, D. M. Greenblatt, E. C. Meng, and T.E. Ferrin (2004). UCSF Chimera-A visualization system for exploratory research and analysis. J. Comput. Chem. 25: 1605-1612.

Plett, D., G. Safwat, M. Gilliham, I.S. Møller, S. Roy, N. Shirley, A. Jacobs, A. Johnson, and M. Tester (2010). Improved salinity tolerance of rice through cell type-specific expression of AtHTK1;1. PLoS ONE. 5.

Quan, R., H. Lin, I. Mendoza, Y. Zhang, W. Cao, Y. Yang, M. Shang, S. Chen, J.M. Pardo, and Y. Guo (2007). SCABP8/CBL10, a putative calcium sensor, interacts with the protein kinase SOS2 to protect Arabidopsis shoots from salt stress. Plant cell. 19(4): 1415-1431.

Quintero, F. J., J. Martinez-Atienza, I. Villalta, X. Jiang, W. Y. Kim, Z. Ali, H. Fujii, I. Mendoza, D.J. Yun, J.K. Zhu, and J. M. Pardo (2011). Activation of the plasma membrane $\mathrm{Na} / \mathrm{H}$ antiporter Salt-Overly-Sensitive 1 (SOS1) by phosphorylation of an auto-inhibitory C-terminal domain. Proc. Natl. Acad. Sci. U.S.A., 108(6): 2611-2616.

Qiu, Q. S., Y. Guo, M. A. Dietrich, K. S. Schumaker, and J. K. Zhu (2002). Regulation of SOS1, a plasma membrane $\mathrm{Na}+\mathrm{H}+$ exchanger in Arabidopsis thaliana, by SOS2 and SOS3. Proc. Natl. Acad. Sci. U.S.A., 99(12): 8436-8441.

Ren, Z.H., J.P. Gao, L.G. Li, X.L. Cai, W. Huang, D.Y. Chao, M.Z. Zhu, Z.Y. Wang, S. Luan, and H.X. Lin (2005). A rice quantitative trait locus for salt tolerance encodes a sodium transporter. Nat. Genet. 37: 1141-1146.

Roy, S.T., S. Negrão, and M. Tester (2014). Salt resistant crop plants. Curr. Opin. Biotechnol. 26: $115-$ 124.

Rodríguez-Rosales, M.P., F.J. Gálvez, R. Huertas, M.N. Aranda, M. Baghour, O. Cagnac, and K. Venema (2009). Plant NHX cation/proton antiporters. Plant Signal Behav. 4(4): 265-76.

Rushmore, T. H., M. R. Morton, and C. B. Pickett (1991). The antioxidant responsive element. Activation by oxidative stress and identification of the DNA consensus sequence required for functional activity. J. Biol. Chem. 266: 11632 11639. 
Shi, H., M. Ishitani, C. Kim, and J. K. Zhu (2000). The Arabidopsis thaliana salt tolerance gene SOS1 encodes a putative $\mathrm{Na}+/ \mathrm{H}+$ antiporter. Proc. Natl. Acad. Sci., 97(12): 6896-6901.

Srivastava, A.K., C. Zhang, G. Yates, M. Bailey, A. Brown, and A. Sadanandom (2016). SUMO is a critical regulator of salt stress responses in rice. Plant Physiol. 170(4): 2378-2391.

Sun, J., H. Cao, J. Cheng, X. He, H. Sohai, M. Niu, Y. Huang, and Z. Bie (2018). Pumpkin CmHKT1;1 controls shoot $\mathrm{Na}^{+}$accumulation via limiting $\mathrm{Na}^{+}$transport from rootstock to scion in grafted cucumber. Int. J. Mol. Sci. 19(9): 2648-2668.

Sunarpi., T. Horie, J. Motoda, M. Kubo, H. Yang, K. Yoda, R. Horie, W.Y. Chan, H.Y. Leung, K. Hattori, M. Konomi, M. Osumi, M. Yamagami, J. I. Schroeder, and N. Uozumi (2005). Enhanced salt tolerance mediated by AtHKT1 transporter-induced $\mathrm{Na}+$ unloading from xylem vessels to xylem parenchyma cells. Plant J. 4: 928-938.

Suzuki, K., N. Yamaji, A. Costa, E. Okuma, N.I. Kobayashi, T. Kashiwagi, M. Katsuhara, C. Wang, K. Tanoi, Y. Murata, J.I. Schroeder, J.F. Ma, and T. Horie (2016). OsHKT1;4-mediated $\mathrm{Na}+$ transport in stems contributes to $\mathrm{Na}+$ exclusion from leaf blades of rice at the reproductive growth stage upon salt stress. BMC Plant Biol. 16: 22.
Tester, M., and R. Davenport (2003). $\mathrm{Na}^{+}$tolerance and $\mathrm{Na}^{+}$transport in higher plants. Ann. Bot. 91: 503-527.

Wang, R., W. Jing, L. Xiao, Jin Y. Jin, L. Shen, and W. Zhang (2015). The rice high-affinity potassium transporter 1;1 is involved in salt tolerance and regulated by an MYB-type transcription factor. Plant Physiol. 168: 1076-1090.

Whiteman, SA., T.S. Nuhse, D.A. Ashford, D. Sanders, and F.J. Maathuis (2008). A proteomic and phosphoproteomic analysis of Oryza sativa plasma membrane and vacuolar membrane. Plant J. 56: 146-156.

Yanagisawa, S., and R. J. Schmidt (1999). Diversity and similarity among recognition sequences of Dof transcription factors. Plant J. 17: 209-214.

Yao, X., T. Horie, S. Xue, H.Y. Leung, M. Katsuhara, D.E. Brodsky, Y. Wu, and J.I. Schroeder (2010). Differential sodium and potassium transport selectivities of the rice OsHKT2;1 and OsHKT2;2 transporters in plant cells. Plant Physiol. 152(1): 341-55.

Zhang, Y. (2008). I-TASSER server for protein 3D structure prediction. BMC Bioinformatics. 9: 40.

Zhang, Z.L., Z. Xie, X. Zou, J. Casaretto, T.H. Ho, and Q.J. Shen (2004). A rice WRKY gene encodes a transcriptional repressor of the gibberellin signaling pathway in aleurone cells. Plant Physiol. 134: 1500-1513. 